\title{
Design of hydrogen transmission pipeline networks with hydraulics
}

\author{
Alexandra C. Weber ${ }^{a, b}$, Lazaros G. Papageorgiou ${ }^{b}$ \\ a Aachener Verfahrenstechnik - Process Systems Engineering, RWTH \\ Aachen University, Forckenbeckstraße 51, 52074 Aachen, Germany \\ ${ }^{b}$ Centre for Process Systems Engineering, University College London (UCL), \\ London WC1E 7JE, United Kingdom
}

\begin{abstract}
In order to enable a more sustainable transport sector in the future, a mixedinteger linear programming (MILP) model is developed with the aim of designing a pipeline network for hydrogen transmission. The objective of the optimisation is the minimisation of the network cost while taking hydraulics into consideration. Relevant features, i.e. maximum flow rate and facility location problem are included. Furthermore, the objective of pipeline safety is investigated based on an index-based risk assessment by Kim and Moon [1]. To examine the capabilities of the developed model, a case study on Germany is conducted for several scenarios. The optimised networks are discussed and compared. A Pareto frontier is computed in order to study the trade-off between network cost and safety.
\end{abstract}

Keywords: hydrogen pipeline networks; hydraulics; MILP; multi-objective Optimisation

\section{Introduction}

As the concept of sustainability for future applications in the energy and transport sector is gaining importance, the interest in hydrogen as an alternative to fossil fuels is increasing. With its versatility concerning production, storage and transport technologies, as well as its function as a low emission energy-carrier, hydrogen offers itself to ensure energy security in a society 
relying on regenerative resources. Due to the continual research and improvement on fuel cell vehicles (FCV) [2], hydrogen will be especially beneficial in the transport sector.

To this end, a hydrogen supply chain (HSC) has to be put into place to secure hydrogen availability and promote popularity of FCV. The so-called 'chicken-and-egg'-dilemma [3] outlines the difficulties that are connected to the construction of an extensive HSC: companies involved in hydrogen production are reluctant to invest into the HSC without prospects of profit, while hydrogen consumers will hesitate to buy FCV as long as the infrastructure is lacking. It is, therefore, of interest to both parties, that research is conducted on the HSC and its individual components to enable a smooth transition into a hydrogen-fuelled society. The individual components of a HSC consist of hydrogen production, storage and transportation technologies. For the latter, distribution and transmission of hydrogen can be distinguished. The physical state of hydrogen is vital for the chosen transportation mode. Liquid hydrogen is, i.e. transported by cryogenic trucks, gaseous hydrogen by tube trailers or pipelines $[4,5]$. Hydrogen production technologies range from highly emission-intensive, i.e., coal gasification and steam methane reforming (SMR), to carbon-neutral ones, i.e., biomass gasification and water electrolysis $[6,7]$. The former technologies offer themselves to centralised hydrogen production scenarios, but should be combined with carbon capture and storage (CCS) to ensure sustainability. Water electrolysis is expected to be of interest in the future due to its modular nature and flexibility concerning the start-up process.

Mathematical models on HSC investigating a selection of hydrogen technologies have been discussed in literature many times over [8, 9, 10, 11, 12]. Some of the earliest research has been done by Almansoori and Shah [13], and a lot of models are based on their work or contain some components [6, $8,9,10,11,12,14,15,16,17,18]$. Apart from the technologies which are included, these works differ in their objective. Some of them evaluate the network profit [14], while most concentrate on minimising the investment cost $[9,12,13,18]$. Furthermore, multi-periodity is investigated $[9,16,18]$. While most of the aforementioned works define the hydrogen demand over a grid, several studies apply geographical information systems (GIS) in order to enable a more detailed analysis of the demand distribution [19, 20].

Multi-objectivity is another much researched topic in HSC. The most favoured objective is the economic cost of the HSC. Other objectives in literature are environmental impact $[6,10,21,22,23]$ and safety $[1,8,24]$. For the former, several approaches have been developed. Firstly, principles of Life Cycle Assessment (LCA) have been used by Guillen-Gosálbez et al. [10] 
and Sabio et al. [6]. Secondly, Almansoori and Betancourt-Torcat defined the environmental impact by carbon-dioxide emission [23]. For safety considerations, a risk assessment has been developed and applied by Kim and Moon [24]. Finally, multi-objective works considering all three objectives have been conducted by Han et al. and Almaraz et al. [8, 14, 25, 26].

Little research has been conducted on hydrogen pipelines, despite them being suitable to transport large volumes of hydrogen over large distances [27]. Johnson and Ogden have developed a model that allows the optimisation of interconnected pipeline networks [28]. Baufumé et al. investigate scenarios for a pipeline network for Germany combined with GIS [20] and André et al. develop an local search method optimising both the network design and dimensioning [29].

In the scope of this work, a mixed-integer linear programming (MILP) model for the optimisation of a pipeline network in Germany is developed based on the non-linear programming (NLP) model by André et al. The detailed formulation of the MILP model is presented in Section 3. The mathematical model formulations allow the minimisation of economic cost while determining the optimal pipeline network design and diameter of the pipe segments. The implementation of the facility location problem into the model is described in three scenarios. Furthermore, the mathematical formulations are extended to a multi-objective problem. The case study on Germany is presented in Section 4. Then, in Section 5, the results for each scenario are discussed and, if relevant, compared to each other. Finally, Section 6 gives some concluding remarks and suggests future work.

\section{Problem statement}

The goal of this work is the design of an optimised hydrogen pipeline network. This includes the determination of the most advantageous pipeline connections, but also their dimensions. To this end, the locations of demand centres as well as their individual hydrogen demand are given. The connections between the nodes represent potential pipeline arcs; the distances that they span are defined as the delivery distance and by that as the length of a potential pipeline. The pipeline dimensions are chosen from a set of discrete diameters.

In addition to the goal of designing the optimal pipeline network and its dimensions, other factors of interest are the flow rates of hydrogen inside of the pipelines, as well as the pressure losses occurring over delivery distances. Hydraulics of the pipeline are considered to obtain this information. Interna- 
tional import of hydrogen is enabled and the amount of hydrogen imported in a network is determined. Furthermore, the optimal location of the supply nodes is investigated.

The objective of the model is the minimisation of the total cost linked to the construction and the operation of such a hydrogen pipeline network. Total cost include the annualised investment and maintenance cost for hydrogen pipelines and hydrogen production sites. A fraction of the investment cost is allocated as annual maintenance cost. Furthermore, operational costs are considered in form of the hydrogen production cost.

\section{Mathematical model}

The hydrogen pipeline network problem is based on the NLP formulation by André et al. [29]. By approximation and addition of other features, i.e. a neighbourhood-flow approach and a restriction on the flow rate, an MILP problem formulation is developed. The model is formulated in the General Algebraic Modeling System (GAMS) and solved using CPLEX 12.7.1.0 solver on a $2.6 \mathrm{GHz}, 128 \mathrm{~GB}$ RAM machine (Intel ${ }^{\circledR}$ Xeon ${ }^{\circledR}$ CPU E5-2640 v3).

Objective function The total network cost $T C$ is composed of the pipeline investment and maintenance cost $P C$, the hydrogen production $H C$ and import $I C$ cost as well as the production site $F C$ investment and maintenance cost.

$$
T C=H C+I C+P C+F C
$$

The capital recovery facor $\operatorname{crf}$ converts the overall investment costs to portions that are due annually.

$$
\operatorname{cr} f=\frac{i t(1+i t)^{n}}{(1+i t)^{n}-1}
$$

where $i t$ is the interest rate and $n$ the number of annuities.

The annual maintenance cost of pipelines are estimated based on the pipeline investment cost $C C P$.

$$
M P=m_{p} * C C P
$$

Maintenance cost of production sites are determined in a similar manner. 
Pipeline transmission A graph consisting of a set of nodes $i \in N$ is constructed. The connection between these nodes is denoted as the subset of $\operatorname{arcs} A \in N \times N$. Hydrogen can be transported on all arcs of the subset. The subset $A$ is adjusted with help of an adaption of the neighbourhood-flow approach that has been developed by Akgul et al. [30] (Section A.1.1). The approach limits the subsets of possible pipeline connections to those that connect nodes in neighbouring regions. Exceptions are made for very short arcs.

A mass balance at each node ensures that the demand $d e m_{i}$ is covered by either hydrogen supply directly at the node $s E_{i}$, hydrogen import $\hat{I}_{i}$ or flows from other nodes $\sum_{j \mid(j, i) \in A} Q_{j i}$ :

$$
s E_{i}+\hat{I}_{i}+\sum_{j \mid(j, i) \in A} Q_{j i}=d e m_{i}+\sum_{j \mid(i, j) \in A} Q_{i j}, \forall i \in N
$$

At the same time, the overall hydrogen demand of the network $M_{S}$ is provided by both hydrogen supply and import. No surplus hydrogen is allowed to be produced or imported.

$$
\sum_{i \in N} s E_{i}+\sum_{i \in N} \hat{I}_{i} \leq M_{S}
$$

The hydrogen flow on the inside of the pipelines is modelled by a linearised form of the general flow equation [31]. For approximation, the square of the pressures at individual nodes is defined as a continuous variable $\pi_{i}$ and a set of discrete diameters $d \in D$ are introduced. Piecewise approximation is applied to the bilinear pressure term $\sqrt{\pi_{i}-\pi_{j}} Y_{d i j} \equiv \theta Y_{d i j}$. Details on the equations for piecewise approximation can be found in the Appendix, Section A.1.2.

$$
\sum_{d \in D} \hat{d}_{d}^{2.5} \theta Y_{d i j}=k_{p l} L_{i j}{ }^{0.5} Q_{i j}, \forall(i, j) \in A
$$

The binary variable $Y_{d i j}$ allows the pipeline $\operatorname{arcs}$ of the subset $A$ to be chosen according to the active pipeline network. Although the subset of arcs $A$ allows for bi-directionality, the flow between two nodes is restricted to a single direction.

$$
\sum_{d \in D} Y_{d i j}+Y_{d j i} \leq 1, \forall(i, j) \in A
$$


Depending on the diameter $\hat{d}_{d}$ that is chosen for the active pipeline arc, the flow rate $Q_{i j}$ is restricted by the maximum flow rate $k Q_{d}^{\text {max }}$ associated with the average pressure $\psi Y_{d i j}$ in the pipeline.

$$
Q_{i j} \leq \sum_{d \in D} k Q_{d}^{\max } \psi Y_{d i j}, \forall(i, j) \in A
$$

Parameter $k Q_{d}^{\max }$ connects the flow rate at standard conditions $Q_{i j}$ to the flow rate at active pipeline conditions $Q_{a}$ by the equation of continuity. By this, velocities in the pipeline are restricted to $30 \mathrm{~m} \mathrm{~s}^{-1}$ [3]. For the determination of the average pressure in the pipeline, a simple approach is chosen:

$$
\bar{\pi}_{i j}=\sqrt{0.5\left(\pi_{i}+\pi_{j}\right)}
$$

This approach yields similar values as the equation commonly found in literature [31] and, at the same time, can easily be approximated by piecewise linearisation, similarly to the aforementioned pressure difference. To this end, the continuous variable $\psi Y_{d i j} \equiv \sqrt{0.5\left(\pi_{i}+\pi_{j}\right)} Y_{d i j}$ is introduced for the bi-linear term of the average pressure. Again, the approach is detailed in the Appendix (Section A.1.3.).

Another constraint is introduced, restricting the flow rates further. By doing so, Equation 3.4 is bound to its feasible region.

$$
Q_{i j} \leq \sum_{d \in D} Q_{d}^{\max } Y_{d i j}, \forall(i, j) \in A
$$

Here, $Q_{d}^{\max }$ is the maximum flow rate allowed by the general flow equation. Finally, the range of the squared pressures is defined by adding an upper and lower bound.

$$
\pi^{\text {min }} \leq \pi_{i} \leq \pi^{\max }, \forall i \in N
$$

Both the diameter $\hat{d}_{d}$ and the length $L_{i j}$ of a pipeline affect its cost. As the investment cost of pipelines is a quadratic function, the diameter has a more distinct influence. The investment cost for pipelines is based on the equations by Yang and Ogden [32] but linearised by discretisation of the diameter.

$$
P C=\left(\operatorname{crf}+m_{p}\right) \sum_{(i, j) \in A} L_{i j} \sum_{d \in D}\left(a_{0}+a_{12 d}\right) Y_{d i j}
$$

where $a_{0}$ and $a_{12 d}$ are the cost coefficients and $m_{p}$ is the percentage of the investment cost allocated to annual maintenance. 
Facility location The facility location problem is treated in three scenarios: A) the supply node is arbitrarily defined (base scenario), B) The location of a single supply node is optimised, and $C$ ) production sites are chosen with respect to available capacities. In the last scenario, SMR is chosen as a production technology. Each scenario brings the network model closer to reality, but at the same time, the importance of an ideal pipeline network design decreases. It has to be mentioned that all of the nodes $i$ are considered as possible hydrogen production sites for scenario two and three. Other factors, i.e., the availability of suitable sites for the construction of production facilities, are neglected.

The maximum hydrogen supply is restricted to the overall hydrogen demand for all three scenarios.

$$
s E_{i} \leq M_{S} E 1_{i}, \forall i \in N
$$

This definition prevents surplus production of hydrogen and at the same time introduces the binary variable $E 1_{i}$, which chooses the active supply nodes from the set of available ones. For each active supply node, the pressure at which hydrogen is supplied is restricted.

$$
\pi_{i} \geq \pi^{\max } E 1_{i}, \forall i \in N
$$

It is assumed that hydrogen is supplied at maximum pressure $\pi^{\max }=$ $\left(p^{\max }\right)^{2}$. Again, the pressure bound applies only for active supply nodes, which is achieved by the use of the binary variable $E 1_{i}$. The greater sign in Equation 3.11 has been chosen due to the necessity of enabling pressures greater than zero for those nodes that are not used for hydrogen supply. Combining this inequality equation with Equation 3.8, the pressure at node $i$ is determined as the maximum pressure at supply nodes, while for all other cases the pressure is allowed to vary between minimum and maximum pressure as computed by the application of the pressure loss equation (see Equation 3.4).

For scenario $A$ ), $E 1_{i}$ is defined according to the desired production node. Scenario $B$ ) with one optimised supply node requires an additional constraint regarding the number of constructed supply nodes. This definition allows only one supply node to be activated.

$$
\sum_{i \in N} E 1_{i} \leq 1
$$

Lastly, scenario $C$ ) requires the extension of the binary variable $E 1_{i}$ by the set of capacities $k \in K$. A new binary variable $E_{k i}$ is therefore introduced 
and Equations (3.10) to (3.12) are adjusted accordingly:

$$
s E_{i} \leq \sum_{k \in K} A_{k} E_{k i}, \forall i \in N
$$

where the parameter $A_{k}$ defines the capacities of each production plant size.

$$
\begin{gathered}
\pi_{i} \geq \pi^{\max } \sum_{k \in K} E_{k i}, \forall i \in N \\
\sum_{i \in N} \sum_{k \in K} E_{k i} \leq 1
\end{gathered}
$$

Equation 3.14 is responsible for defining the pressure at which hydrogen is supplied, while Equation 3.15 is altered slightly, the adjustment permitting one production site of a certain capacity per node.

The cost coefficients for hydrogen production and import are introduced as parameters $c H$ and $i p$, respectively. Accordingly, the annual cost of hydrogen production is defined as follows:

$$
H C=c H \sum_{i \in N} s E_{i}
$$

In the same manner, hydrogen import cost depends on the volume of hydrogen that is imported from other countries.

$$
I C=i p \sum_{i \in N} \hat{I}_{i}
$$

The investment and maintenance cost for the construction of hydrogen production sites is included in scenario $C$ ). As before, the annual maintenance cost is assumed to be a fraction $m_{f}$ of the overall investment cost.

$$
F C=\left(c r f+m_{f}\right) \sum_{i \in N} \sum_{k \in K} c c A_{k} E_{k i}
$$

Safety objective As an additional objective, the safety of the pipeline network is of interest, especially because of the high combustibility of hydrogen as compared to conventional fuels. The index-based risk assessment developed by Kim et al. is, therefore, implemented for the pipeline network [1].

According to Kim et al., the total relative risk of a hydrogen operation depends on the inherent risk factor (IRF) of the specific operation and the 
environmental impact factor (EIF) which describes the safety of its location. Since pipelines connect different regions, the total relative risk index (TRRI) depends on the safety of the starting and ending region, as well as the intermediate ones.

$$
T R R I=\sum_{(i, j) \in A} I R F E I F_{i j}
$$

The epsilon-constraint method is applied as a solution strategy for the multi-objective model version [33]. The method converts the safety objective into a constraint and minimises the annualised cost for different upper bounds on the TRRI. This way, the different Pareto-optimal solutions, each of them composing an individual hydrogen network design, are computed. The possible configurations are then visualised in the Pareto frontier, from which the most promising design can be chosen depending on preference of the developer.

Model summary Three optimisation problems can be derived from the formulations for facility location, as defined by the labels $A$ ) to $C$ ). The model equations for each scenario are summarized in the following:

Scenario A)

$\min T C$

s.t. $\left\{\begin{array}{l}\text { Demand constraints (3.2) } \\ \text { Production constraints (3.10) and (3.11) } \\ \text { Import constraints (3.3) } \\ \text { Hydraulics (3.4) to (3.8) and (A.1) to (A.10) }\end{array}\right.$

$\min T C$

Scenario $B$ )

s.t. $\left\{\begin{array}{l}\text { Demand constraints }(3.2) \\ \text { Production constraints (3.10) to (3.12) } \\ \text { Import constraints (3.3) } \\ \text { Hydraulics (3.4) to (3.8) and (A.1) to (A.10) }\end{array}\right.$




$\begin{array}{ll}\text { Scenario } C) & \text { min } \\ \text { s.t. }\left\{\begin{array}{l}\text { Demand constraints (3.2) } \\ \text { Production constraints (3.10), (3.14) and (3.15) } \\ \text { Import constraints (3.3) } \\ \text { Hydraulics (3.4) to (3.8) and (A.1) to (A.10). }\end{array}\right.\end{array}$

Each of the scenarios can be extended to a multi-objective optimisation problem by including the epsilon-constraint of the safety objective, as presented with the example of scenario $A$ ).

$\begin{array}{ll}\min & T C \\ \text { Multi-objective } & \text { s.t. }\left\{\begin{array}{l}T R R I \leq \epsilon \\ \text { Demand constraints (3.2) } \\ \text { Production constraints (3.10) and (3.11) } \\ \text { Import constraints (3.3) } \\ \text { Hydraulics (3.4) to (3.8) and (A.1) to (A.10) }\end{array}\right.\end{array}$

\section{Case study}

A case study is performed on Germany to put the different model versions to test. In Germany, a great interest in alternative fuels is given in the energy and transport sector. This is closely connected to politics, but also greatly supported by the population. One of the aims pursued by politics, i.e., is to shut down all nuclear power plants until 2022 [34]. Another goal envisaged until 2050 are zero emissions [23]. The NUTS-1 resolution consisting of the 16 states of Germany is applied for each scenario the case study. For each state, the city with the largest population functions as a node.

Demand assumptions For the determination of the hydrogen demand of the individual states, a market scenario developed in the scope of Hyways project, a European consortium striving for a sustainable future with help of hydrogen [35], is chosen. With the assumption of high policy support and a modest learning curve, the market penetration rate of hydrogen is set to $40 \%$. Based on this penetration rate, a hydrogen demand for the transportation sector is determined 


$$
\operatorname{dem}_{i}=\frac{1}{\rho_{b}} P T P_{i} V O_{i} C U f c
$$

where $d^{2} m_{i}$ is the demand, $P T$ the market penetration rate, $P_{i}$ the population of each region, $V O_{i}$ is the vehicle ownership in each region, $C U$ the car use per year and $f c$ the fuel consumption. A similar approach is frequently applied in literature $[7,8,13,36]$. The hydrogen demand determined for each German state is presented in Table 4.1.

\begin{tabular}{lllcc}
\hline Nuts code & Name of state & Largest city & $\begin{array}{c}\text { Population } \\
{\left[10^{6}\right]}\end{array}$ & $\begin{array}{c}\mathrm{H}_{2} \text { demand } \\
{\left[10^{3} \mathrm{~m}^{3} \mathrm{hr}^{-1}\right]}\end{array}$ \\
\hline DE1 & Baden-Wurttemberg & Stuttgart & 10.5 & 376.5 \\
DE2 & Bavaria & Munich & 12.4 & 452.5 \\
DE3 & Berlin & Berlin & 3.3 & 69.1 \\
DE4 & Brandenburg & Potsdam & 2.5 & 83.5 \\
DE5 & Bremen & Bremen & 0.7 & 17.1 \\
DE6 & Hamburg & Hamburg & 1.7 & 45.4 \\
DE7 & Hesse & Frankfurt & 6.0 & 212.8 \\
DE8 & Mecklenburg-Western Pomerania & Rostock & 1.6 & 51.2 \\
DE9 & Lower Saxony & Hanover & 7.8 & 275.1 \\
DEA & North Rhine-Westphalia & Cologne & 17.5 & 586.6 \\
DEB & Rhineland-Palatinate & Mainz & 4.0 & 146.6 \\
DEC & Saarland & Saarbrücken & 1.0 & 37.9 \\
DED & Saxony & Dresden & 4.1 & 128.8 \\
DEE & Saxony-Anhalt & Magdeburg & 2.3 & 74.3 \\
DEF & Schleswig-Holstein & Kiel & 2.8 & 95.7 \\
DEG & Thuringia & Erfurt & 2.2 & 72.1 \\
Total & & & 80.4 & 2725.2 \\
\hline
\end{tabular}

Table 4.1: Population and hydrogen demand of the 16 German states.

Pipeline transmission Standard conditions are defined for the consideration of hydrogen transmission. These conditions, as well as necessary physical properties of hydrogen and other parameters are presented in the Appendix (Table A.2). Other data, i.e. the defined discrete diameters of pipelines, delivery distances between nodes and bounds for pressure, is included in Tables A.2 to A.6. Tables A.7 and A.8 summarise the pipeline data 
for safety investigations. According to the investigations of Kim et al., a risk level of IV is assigned for hydrogen transport via pipelines [24]. A risk level of IV implies several casualties in case of an accident occurring on a section of a hydrogen pipeline, as well as a negative impact on the environment of one to three years. Additionally, an interruption to the hydrogen transport occurs for several months.

Production plants As the problem formulation assumes a centralised hydrogen production scenario, SMR is chosen as a suitable technology. Three sizes of SMR plants are defined to appoint capacities, as presented in Table A.11.

\section{Results and discussion}

The aim of the proposed model is to design a hydrogen network with optimised diameter sizes, while satisfying the hydrogen demand in Germany. The solutions obtained by optimising the scenarios described in the model summary of Section 3 are presented. In the scope of the case study on Germany, the scenarios are defined as follows.

- Scenario A) (Section 5.1): Berlin (DE3) as single supply node

- Scenario B) (Section 5.2): Optimal location of single supply node

- Scenario C) (Section 5.3): Multiple production facilities

Additionally, each scenario is treated as a multi-objective optimisation problem, as described in Section 5.4.

\subsection{Scenario $A$}

Figure 5.1 presents the optimised network for the base scenario, which assumes Berlin (DE3), the capital of Germany, as a supply node. The data, i.e., the pipe diameters and flow rates determined by optimisation, are given in Table A.13: from Berlin, pipelines of diameters between $25 \mathrm{~cm}$ and $100 \mathrm{~cm}$ transmit hydrogen to all other parts of Germany. The largest flow of hydrogen is given in the pipeline connection between the supply node DE3 and its nearest neighbour Potsdam (DE4). With a flow rate of $2,656,217.7 \mathrm{~m}^{3} \mathrm{hr}^{-1}$ at standard conditions, only a pipeline of $100 \mathrm{~cm}$ is able to transport this large volume of hydrogen. From DE4 on, a pipeline branch stretches out in 
the direction of south-west. Reason for this is the large demand accumulating in that area. At Frankfurt am Main (DE7), the pipeline branch is split to provide hydrogen to the west and south of Germany. The overall

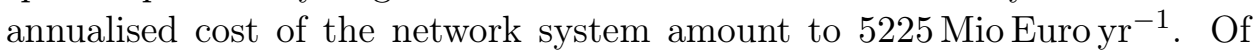
this sum, 4775 Mio Euro $\mathrm{yr}^{-1}$ is allocated to hydrogen production. Rather high investment cost of 306 Mio Euro $\mathrm{yr}^{-1}$ for the pipeline system accrue due to large diameters of relevant pipeline arcs.

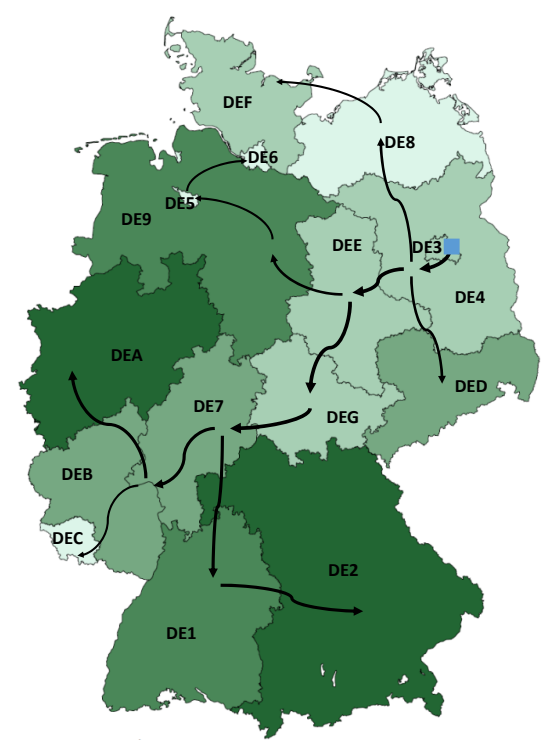

Figure 5.1: Pipeline network design for supply node at DE3 (ם).

The importance of pipeline diameter is revealed when comparing the network design of this work with the minimal spanning tree problem. In regard to delivery distance, little difference exists between both options, amounting to $1910.5 \mathrm{~km}$ for the base scenario and $1797.8 \mathrm{~km}$ for the minimal spanning tree system. This makes the gap in overall pipeline investment cost all the more surprising. While the network configuration designed by this work's model demands investments of overall 2959 Mio Euro into the pipeline system, the cost amount to 3231 Mio Euro for the shortest possible network design. The difference in overall cost can be explained by the diameters of the 
pipelines that are chosen for both network configurations. By minimising the objective cost, this work's model allows for an increase in delivery distance in order to decrease pipe diameters. This way, the capacity defined for the pipe diameters can be exploited for each pipeline segment of the network design.

\subsection{Scenario $B$}

As the distribution of demand already indicates, DE4 is not the best choice as a supply node. The eastern states of Germany have fewer inhabitants than the west and, therefore, demand of hydrogen for light-duty vehicles is lower. To locate the optimal supply node, scenario $B$ ) is investigated. The resulting pipeline network is presented in Figure 5.2. DE7 is identified as the optimal supply node for the given distribution of hydrogen demand. The city offers itself as a supply node due to its location in the centre of Germany,allowing for short delivery distances to cover the high demands of hydrogen in the south-west. Especially North-Rhine Westphalia (DEA) and Lower Saxony (DE9) in the north of DE7 as well as Baden-Wuerttemberg (DE1) and Bavaria (DE2) in the south stand out in terms of hydrogen demands. Those four regions are the ones with the highest hydrogen demands in overall Germany. From DE7 on, four separate pipeline branches spread out to cover the demand at all nodes. Each pipeline branch provides hydrogen for the north, east, south and west of Germany.

In comparison to base scenario, the annualised cost for the hydrogen network is reduced to 5084 Mio Euro $\mathrm{yr}^{-1}$ for scenario B). This reduction by 140 Mio Euro $\mathrm{yr}^{-1}$ comes about as a result of the decreased volumes of hydrogen transported by pipelines, as presented in Table A.14. Pipeline diameters of $75 \mathrm{~cm}$ or less suffice for the four pipeline branches, while four pipelines of $100 \mathrm{~cm}$ are necessary for the base scenario (Table A.13). In contrast to the pipeline diameter, the length of the pipeline network for hydrogen delivery plays an insignificant role. Despite an additional pipeline length of about $120 \mathrm{~km}$ as compared to the base scenario, the overall pipeline investment cost of scenario B's network design is reduced.

\subsection{Scenario $C$}

With regard to capacities of SMR plants, the location of hydrogen supply nodes and the effect of their position on the network design are investigated in Scenario $C$ ).

The results are presented in Figure 5.3. Six production plants of the 


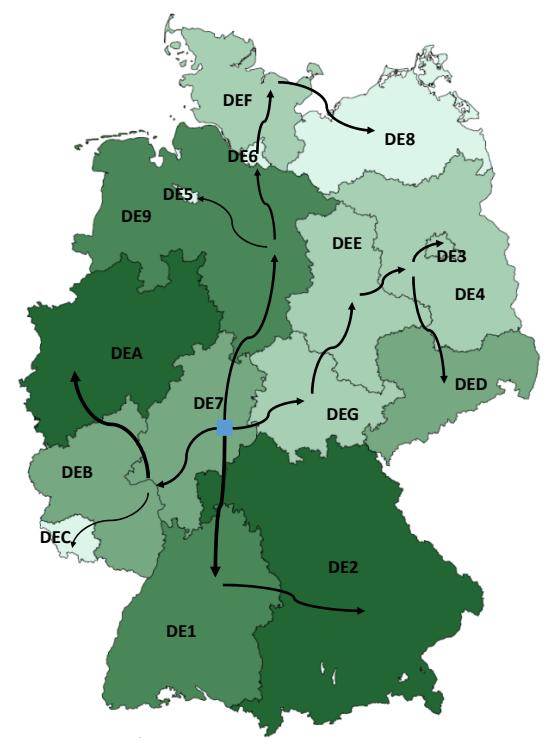

Figure 5.2: Pipeline network design for the MILP problem with location of optimal supply node (ם).

largest possible size are built to satisfy the hydrogen demand, as presented in Tabel A.15. With hydrogen production rates between 376 to $50010^{3} \mathrm{~m}^{3} \mathrm{hr}^{-1}$, all of the production plants utilise a large part to all of their capacity. It has to be noticed that four of the six hydrogen plants chosen during optimisation are located in the south-west, while only two plants supply the whole of the northern part of Germany. Pipelines are applied for the transmission of small volumes of hydrogen with the exception of the pipeline supplying DEA with hydrogen.

The overall annualised cost of the pipeline network amounts to 5480 Mio Euro $\mathrm{yr}^{-1}$. A large part of this sum can be allotted to the investment and maintenance cost of production plants, which are determined with help of a cost analysis (Table A.12). This cost, at least in theory, would also accrue for the other scenarios, since the hydrogen which is transported has to be produced somewhere at some point. For comparison of the three scenarios, the investment cost of the pipeline network is therefore consulted. For the base sce- 


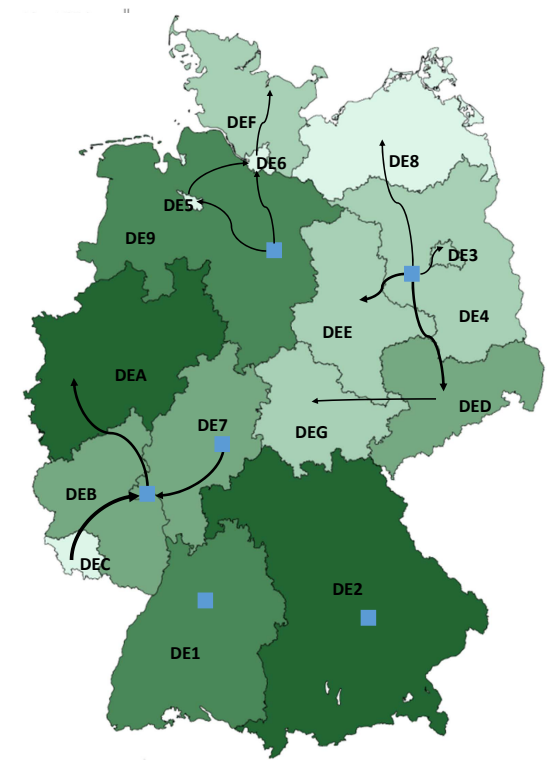

Figure 5.3: Pipeline network design for the MILP problem with production facility location $(\mathbf{\square})$.

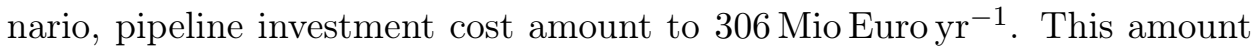
is reduced to 209 Mio Euro $\mathrm{yr}^{-1}$ by locating the optimal supply node in scenario $B$ ). Finally, by allowing several supply nodes, the number of necessary pipeline connections, and with them the cost of the pipeline system can further be reduced to 100 Mio Euro $\mathrm{yr}^{-1}$.

A special feature of the pipeline network design of scenario $C$ ) is the import of hydrogen at DE7. A volumetric flow rate of $8034.9 \mathrm{~m}^{3} \mathrm{hr}^{-1}$ is imported, in addition to the $50010^{3} \mathrm{~m}^{3} \mathrm{hr}^{-1}$ produced at the specific node. The import of this volume is reasonable due to the definition of the production capacities. With the capacity of the smallest available production plant being $4000 \mathrm{~m}^{3} \mathrm{hr}^{-1}$ and that of the medium plant being $7010^{3} \mathrm{~m}^{3} \mathrm{hr}^{-1}$, only the latter would be able to produce the necessary amount of hydrogen. However, the construction of this plant would lead to a higher cost than the import of the amount of hydrogen necessary to satisfy the demand. 


\subsection{Multi-objective}

An investigation of the trade-off between economic cost and pipeline safety is conducted for all three scenarios $A$ ) to $C$ ) The Pareto frontiers of the three scenarios $A$ ) to $C$ ) are presented in Figure 5.4. All Pareto-optimal solutions are computed by variation of the epsilon-variable in 20 equidistant steps to investigate the trade-off between economic cost and pipeline safety.

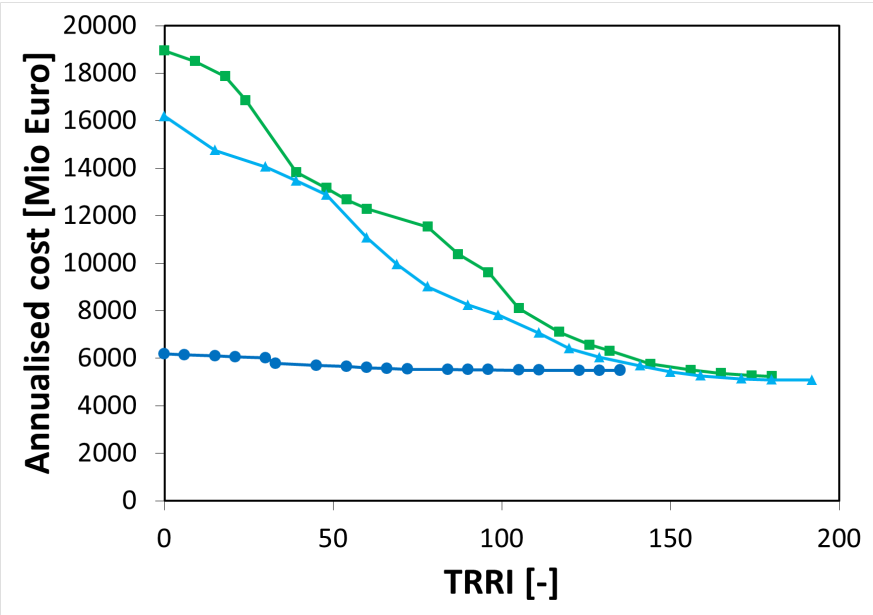

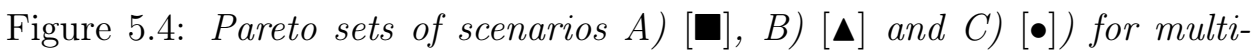
objective problem with cost and safety.

Scenario $A$ ) is connected to high cost for the network design with zero TRRI. For this configuration, the whole of the hydrogen demand is covered by international import. However, with an overall cost of 18,948 Mio Euro yr ${ }^{-1}$, this design is not suitable for realisation. Similarly, scenario $B$ ) gives a network design in which hydrogen is solely imported from other countries. Although the annualised cost of this scenario are lower than for scenario $A$ ), which is triggered by the adjustment of the location of supply, the design is unsuitable due to energy security issues.

The most realistic scenario $C$ ) also achieves the most advantageous network designs. Such a solution is expected due to the possibility of reducing the volume of hydrogen transported by building production plants at several nodes. Furthermore, the TRRI of 147 is achieved by scenario $C$ ), which is the lowest when comparing all three scenarios. The reason for this is the reduced number of pipeline connections necessary to satisfy the demand. Figure 5.5 shows the Pareto set of scenario $C$ ) in finer resolution. As with 
the Pareto frontiers of the other two scenarios, a pronounced drop at a TRRI of 30 indicates the point at which DEA is supplied by pipelines instead of import or hydrogen production at the node. From a TRRI of 72 on, the dependence of TRRI on annualised network cost decreases. This leads to networks which have similar cost but increase drastically regarding network risk. The network design should therefore be chosen from a region between a TRRI of 54 and 90 .

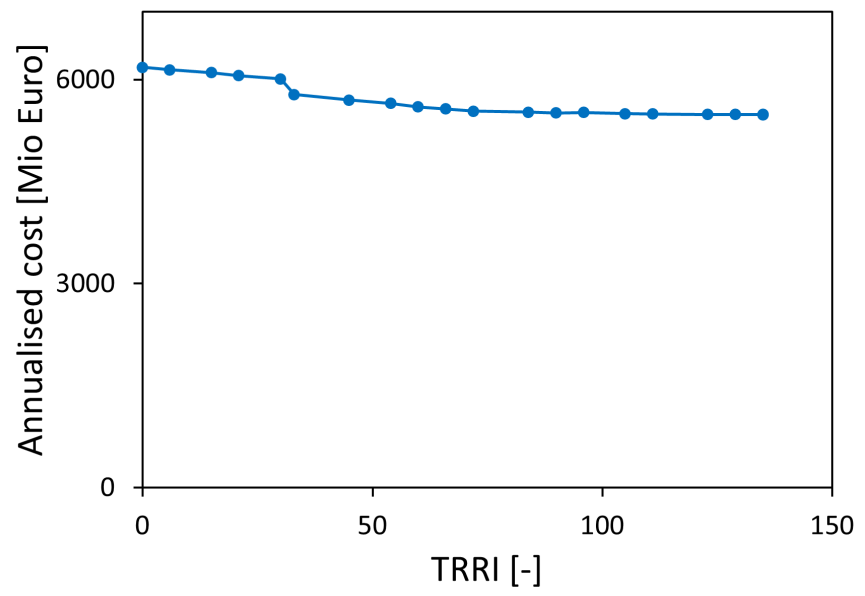

Figure 5.5: Pareto set of scenario C) [•]. for multi-objective problem with cost and safety.

\section{Concluding remarks}

This work has presented an MILP model for the design of a hydraulic pipeline network for hydrogen transmission. Additionally, the facility location problem has been considered in three scenarios. The first scenario has considered an arbitrarily defined node as the supply node; the second has localised the optimal node for hydrogen supply. In the last scenario, the model formulation has been extended to include capacities of hydrogen production plants. Optimisation of economic cost has been defined as the initial objective. Later on, safety of pipeline networks has been added as an objective for all three scenarios.

A case study on Germany has been conducted to investigate the capabilities of each model version. With the supply node located at DE3, large 
volumes of hydrogen have to be transported to the south-west of Germany. The high cost connected to this network design has been reduced by computing the optimal location of the supply node. DE7, which is located between regions with high hydrogen demand, has been found to be the best location for hydrogen production. From there, hydrogen is transported in southern, western, northern and eastern direction in pipelines with diameters smaller than $75 \mathrm{~cm}$. An even lower total cost has been reached for the problem formulation that takes the capacity of production plants into consideration. By restricting the amount of hydrogen that can be produced at each production plant, hydrogen is supplied at several nodes. In turn, the pipeline network is less developed. Although the cost of the overall network design has been reduced, a higher objective than for the other two scenarios has been determined due to the consideration of investment and maintenance cost for production plants.

According to the investigations of the trade-off between network cost and safety for the scenarios $A$ ) to $C$ ), scenario $C$ ) has yielded the most reasonable cost. Scenarios $A$ ) and $B$ ) rely too much on hydrogen import and, on top of that, require more pipeline connections due to the singular supply node. For scenario $C$ ), which has allowed the construction of several production plants, the hydrogen transmission network has been less developed, thus reducing risk posed by the construction and operation of hydrogen pipelines.

In the future, the capabilities of the model should be checked for larger problem sizes. Furthermore, it would be of advantage to extend the facility location problem by relevant factors. Other transport technology, i.e. transport by cryogenic liquid tankers and gaseous trucks, could be included for hydrogen distribution. In addition, compressor stations should be considered to test their impact on the network design.

\section{Acknowledgements}

The authors would like to thank the Aachener Verfahrenstechnik - Process Systems Engineering of the RWTH Aachen University for their support. 


\section{A Appendix}

\section{A.1 Additional details of mathematical model}

\section{A.1.1 Neighbourhood-flow approach}

The computational effort of the problem is reduced by implementing a neighbourhoodflow approach $[18,30]$. It is assumes that only arcs connecting neighbouring regions are to be included into the set of feasible pipeline connections. Connections between nodes that are farther apart and separated by intermediate regions are assumed to be replaceable by a series of connected arcs.

All $\operatorname{arcs}(i, j) \in A$ connecting neighbouring regions are included in the set $A_{1}$. The set is then extended to include very short node connections $A_{2}$, even if the regions in which the nodes are placed are not adjacent. Finally, all long-distanced connections $A_{3}$ are deleted from the set.

$$
\begin{aligned}
& \text { Let }(i, j) \in A_{1}=H, \\
& \text { and }(i, j) \in A_{2} \mid L_{i j} \leq L_{\text {min }}, \\
& \text { and }(i, j) \in A_{3} \mid L_{i j} \geq L_{\max }, \\
& \text { then }(i, j) \in N H=A_{1} \cup A_{2} \cap A_{3} .
\end{aligned}
$$

\section{A.1.2 Piecewise linearisation for pressure difference}

Piecewise approximation of the pressure losses in pipelines allows the linearisation of Equation 3.4. The term $\pi_{i}-\pi_{j}$ is replaced by introducing the SOS2-variable $\lambda_{m 1 i j}$.

$$
\pi_{i}-\pi_{j}=\sum_{m 1 \in M 1} \Delta \hat{\pi}_{m 1} \lambda_{m 1 i j}-s l 1_{i j}, \forall(i, j) \in A
$$

The SOS2-variable chooses the active interval in which the pressure drop is situated from the defined base points $\Delta \hat{\pi}_{m 1}$. To this end, only two consecutive base points $m 1 \in M 1$ of $\lambda_{m 1 i j}$ are activated on each active pipeline arc and restricted to the sum of one by he following equation:

$$
\sum_{m 1 \in M 1} \lambda_{m 1 i j}=\sum_{d \in D} Y_{d i j}, \forall(i, j) \in A
$$

A slack variable $s l 1_{i j}$ ensures that pressure losses are restricted to active pipeline arcs. An additional equation disallows the slack variables from being greater than zero for inactive arcs. 


$$
-M_{\pi}^{\max }\left(1-\sum_{d \in D} Y_{d i j}\right) \leq s l 1_{i j} \leq M_{\pi}^{\max }\left(1-\sum_{d \in D} Y_{d i j}\right), \forall(i, j) \in A
$$

Here, $M_{\pi}^{\max }$ defines the maximum pressure that can be lost through hydrogen transport.

The square root of the pressure loss $\theta Y_{d i j}$ is determined by the so-called function row and with help of the SOS2-variable. Parameter $\hat{\theta}_{m 1}$ quantifies the linear function at the chosen

$$
\sum_{d \in D} \theta Y_{d i j}=\sum_{m 1 \in M 1} \hat{\theta}_{m 1} \lambda_{m 1 i j}, \forall(i, j) \in A
$$

Finally, values of the continuous variable $\theta Y_{d i j}$ are restricted.

$$
\theta Y_{d i j} \leq \hat{\theta}^{\max } Y_{d i j}, \forall(i, j) \in A, d \in D
$$

\section{A.1.3 Piecewise linearization for pressure average}

The average pressure inside of each pipeline arc $0.5\left(\pi_{i}+\pi_{j}\right)$ is approximated by piecewise linearisation, same as the pressure loss over the pipeline arc. The mathematical formulations are similar to those presented in Section A.1.2.

$$
0.5\left(\pi_{i}+\pi_{j}\right)=\sum_{m 2 \in M 2} \hat{\Psi}_{m 2} \tau_{m 2 i j}-s l 2_{i j}, \forall(i, j) \in A
$$

The parameter $\hat{\Psi}_{m 2}$ summarizes all base points used for linearisation, while the SOS2-variable $\tau_{m 2 i j}$ chooses the active interval. Again, only two consecutive base points are allowed to be activated by $\tau_{m 2 i j}$. The SOS2variable is further restricted:

$$
\sum_{m 2 \in M 2} \tau_{m 2 i j}=\sum_{d \in D} Y_{d i j}, \forall(i, j) \in A
$$

A continuous slack variable $s l 2_{i j}$ is introduced to handle pressure differences between nodes that are not connected by a pipeline. The slack variable is restricted to inactive arcs.

$$
\pi^{m i n}\left(1-\sum_{d \in D} Y_{d i j}\right) \leq s l 2_{i j} \leq \pi^{\max }\left(1-\sum_{d \in D} Y_{d i j}\right), \forall(i, j) \in A
$$


Lastly, the average pressure on active pipeline arcs is determined by the function row and bound to the feasible region by Equation A.10.

$$
\begin{gathered}
\hat{\psi}^{m i n} Y_{d i j} \leq \psi Y_{d i j} \leq \hat{\psi}^{m a x} Y_{d i j}, \forall(i, j) \in A, d \in D \\
\sum_{d \in D} \psi Y_{d i j}=\sum_{m 2 \in M 2} \hat{\psi}_{m 2} \tau_{m 2 i j}, \forall(i, j) \in A
\end{gathered}
$$

\section{A.2 Model parameters}

\section{A.2.1 Capital recovery factor and maintenance}

\begin{tabular}{lrr}
\hline Interest rate [\%] & it & 10 \\
Number of annuities [yr] & $n$ & 30 \\
Pipeline maintenance cost percentage [\%] & $m_{p}$ & 5 \\
Production facility maintenance cost percentage [\%] & $m_{f}$ & 5 \\
\hline
\end{tabular}

Table A.1: Cost parameters for capital recovery factor and maintenance cost.

\section{A.2.2 Hydrogen pipeline parameters}

Temperature at standard conditions $[\mathrm{K}]$

273.15

Pressure at standard conditions [bar]

Gas mean temperature $[\mathrm{K}]$

285.15

Molar mass of $\mathrm{H}_{2}\left[\mathrm{~kg} \mathrm{kmol}^{-1}\right]$

Ideal gas constant $\left[\mathrm{kJ} \mathrm{kmol}^{-1} \mathrm{~K}^{-1}\right]$

Isentropic coefficient $\kappa[-]$

Hydrogen compressibility at active conditions [-]

Hydrogen density at standard conditions $\left[\mathrm{kg} \mathrm{m}^{-3}\right]$

Relative density of gas with regard to air (at standard conditions) [-] 0.0596

Table A.2: Definition of standard and active conditions and the corresponding physical properties of hydrogen. 


\begin{tabular}{lcc}
\hline Diameter size d & $\begin{array}{c}\text { Diameter } \hat{d}_{d} \\
{[\mathrm{~cm}]}\end{array}$ & $\begin{array}{c}\text { Maximum flow rate } k Q_{d}^{\text {max }} \\
{\left[10^{-3} \mathrm{~m}^{3} \mathrm{hr}^{-1} \mathrm{bar}^{-1}\right]}\end{array}$ \\
\hline 1 & 25 & 3.84 \\
2 & 50 & 15.36 \\
3 & 75 & 34.55 \\
4 & 100 & 61.43 \\
\hline
\end{tabular}

Table A.3: Discrete diameters and maximum flow rates depending on the average pressure for hydrogen transmission pipelines.

\begin{tabular}{|c|c|c|}
\hline Pipeline installation cost [Mio Euro km $\left.{ }^{-1}\right]$ & $a_{0}$ & $2.8 \times 10^{-1}$ \\
\hline Material cost and others [Mio Euro $\mathrm{km}^{-1} \mathrm{~cm}^{-1}$ ] & $a_{1}$ & $1.29 \times 10^{-4}$ \\
\hline Dependence on diameter [Mio Euro $\mathrm{km}^{-1} \mathrm{~cm}^{-2}$ ] & $a_{2}$ & $2.68 \times 10^{-4}$ \\
\hline
\end{tabular}

Table A.4: Capital cost parameters for pipelines based on Yang and Ogden [32]. 


\begin{tabular}{|c|c|c|c|c|c|c|c|c|c|c|c|c|c|c|c|c|}
\hline & DE1 & DE2 & DE3 & DE4 & DE5 & DE6 & DE7 & DE8 & DE9 & DEA & DEB & DEC & DED & DEE & $\mathrm{DEF}$ & DEG \\
\hline DE1 & & 191 & 512 & 488 & 479 & 535 & 160 & 562 & 402 & 322 & 151 & 167 & 413 & 412 & 621 & 279 \\
\hline DE2 & 191 & & 505 & 486 & 583 & 613 & 326 & 611 & 489 & 487 & 318 & 358 & 360 & 444 & 696 & 319 \\
\hline DE3 & 512 & 505 & & 27 & 316 & 256 & 450 & 182 & 249 & 478 & 454 & 580 & 165 & 128 & 296 & 237 \\
\hline DE4 & 488 & 486 & 27 & & 296 & 242 & 23 & 175 & 225 & 451 & 427 & 553 & 157 & 102 & 289 & 211 \\
\hline DE5 & 479 & 583 & 316 & 296 & & 95 & 336 & 184 & 100 & 248 & 344 & 446 & 406 & 218 & 164 & 279 \\
\hline DE6 & 535 & 613 & 256 & 242 & 95 & & 405 & 94 & 132 & 339 & 412 & 523 & 377 & 193 & 86 & 295 \\
\hline DE7 & 160 & 326 & 450 & 423 & 336 & 405 & & 451 & 276 & 164 & 9 & 130 & 403 & 329 & 489 & 221 \\
\hline DE8 & 562 & 611 & 182 & 175 & 184 & 94 & 451 & & 179 & 413 & 458 & 577 & 327 & 168 & 114 & 296 \\
\hline DE9 & 402 & 489 & 249 & 225 & 100 & 132 & 276 & 179 & & 241 & 283 & 399 & 313 & 132 & 218 & 179 \\
\hline DEA & 322 & 487 & 478 & 451 & 248 & 339 & 164 & 413 & 241 & & 172 & 222 & 486 & 350 & 412 & 298 \\
\hline DEB & 151 & 318 & 454 & 427 & 344 & 412 & 9 & 458 & 283 & 172 & & 125 & 404 & 333 & 497 & 223 \\
\hline DEC & 167 & 358 & 580 & 553 & 446 & 523 & 130 & 577 & 399 & 222 & 125 & & 522 & 459 & 606 & 347 \\
\hline DED & 413 & 360 & 165 & 157 & 406 & 377 & 403 & 327 & 313 & 486 & 404 & 522 & & 188 & 438 & 190 \\
\hline DEE & 412 & 444 & 128 & 102 & 218 & 193 & 329 & 168 & 132 & 350 & 333 & 459 & 188 & & 264 & 135 \\
\hline DEF & 621 & 696 & 296 & 289 & 164 & 86 & 489 & 114 & 218 & 412 & 497 & 606 & 438 & 264 & & 377 \\
\hline DEG & 279 & 319 & 237 & 211 & 279 & 295 & 221 & 296 & 179 & 298 & 223 & 347 & 190 & 135 & 377 & \\
\hline
\end{tabular}

Table A.5: Distance matrix for the largest cities of the 16 German states $[\mathrm{km}]$. 


\begin{tabular}{lll}
\hline Maximum pressure at node $i\left[\mathrm{bar}^{2}\right]$ & $\pi^{\max }$ & 60 \\
Minimum pressure at node $i\left[\mathrm{bar}^{2}\right]$ & $\pi^{\min }$ & 1 \\
\hline
\end{tabular}

Table A.6: Minimum and maximum pressures at nodes.

\begin{tabular}{lc}
\hline Level & Population \\
\hline 1 & $<3 \times 10^{6}$ \\
2 & $3 \times 10^{6}-1 \times 10^{7}$ \\
3 & $>1 \times 10^{7}$ \\
\hline
\end{tabular}

Table A.7: Categorisation scheme for safety levels for nodes.

\begin{tabular}{llcllc}
\hline Region & Size & Safety level & Region & Size & Safety level \\
\hline DE1 & Large & 3 & DE9 & Medium & 2 \\
DE2 & Large & 3 & DEA & Large & 3 \\
DE3 & Medium & 2 & DEB & Medium & 2 \\
DE4 & Small & 1 & DEC & Small & 1 \\
DE5 & Small & 1 & DED & Medium & 2 \\
DE6 & Small & 1 & DEE & Small & 1 \\
DE7 & Medium & 2 & DEF & Small & 1 \\
DE8 & Small & 1 & DEG & Small & 1 \\
\hline
\end{tabular}

Table A.8: Sizes and safety levels of German states for risk assessment.

\begin{tabular}{ll}
\hline$\beta=1$ & A region is crossed by a pipeline \\
$\beta=0.5$ & A pipeline passes near the region \\
\hline
\end{tabular}

Table A.9: Evaluation of the risk of a pipeline connection. 


\begin{tabular}{|c|c|c|c|c|c|c|c|c|c|c|c|c|c|c|c|c|}
\hline & DE1 & DE2 & DE3 & DE4 & DE5 & DE6 & DE7 & DE8 & DE9 & DEA & DEB & DEC & DED & DEE & $\mathrm{DEF}$ & DEG \\
\hline DE1 & 3 & 6 & 10 & 12 & 14 & 11 & 6 & 13.5 & 10 & 10 & 7 & 6 & 8 & 8 & 12 & 7 \\
\hline DE2 & 6 & 3 & 8 & 9 & 9 & 7.5 & 9 & 9 & 7.5 & 13 & 10 & 9 & 5 & 5 & 8.5 & 4 \\
\hline DE3 & 10 & 8 & 1 & 3 & 3 & 6 & 6 & 2 & 4 & 8 & 7 & 8 & 3 & 26 & 3.5 & 3 \\
\hline DE4 & 12 & 9 & 3 & 2 & 7 & 7.5 & 8 & 3 & 6 & 9 & 9 & 10 & 5 & 4 & 5 & 5 \\
\hline DE5 & 14 & 9 & 3 & 7 & 1 & 4 & 9 & 5.5 & 3 & 6 & 8 & 9 & 6 & 4 & 4 & 4 \\
\hline DE6 & 11 & 7.5 & 6 & 7.5 & 4 & 1 & 9 & 3 & 3 & 6 & 10 & 7 & 6.5 & 4 & 2 & 5 \\
\hline DE7 & 6 & 9 & 6 & 8 & 9 & 9 & 2 & 6.5 & 8 & 7 & 4 & 5 & 9 & 5 & 10 & 3 \\
\hline DE8 & 13.5 & 9 & 2 & 3 & 5.5 & 3 & 6.5 & 1 & 3 & 6 & 7 & 11 & 5 & 5 & 2 & 5.5 \\
\hline DE9 & 10 & 7.5 & 4 & 6 & 3 & 3 & 8 & 3 & 2 & 5 & 7 & 10 & 5 & 3 & 4 & 3 \\
\hline DEA & 10 & 13 & 8 & 9 & 6 & 6 & 7 & 6 & 5 & 3 & 7 & 6 & 9 & 7 & 7 & 6 \\
\hline DEB & 7 & 10 & 7 & 9 & 8 & 10 & 4 & 7 & 7 & 7 & 2 & 4 & 10 & 7 & 10.5 & 5 \\
\hline DEC & 12 & 18 & 16 & 20 & 21 & 14 & 10 & 22 & 20 & 12 & 8 & 2 & 22 & 14 & 19 & 11 \\
\hline DED & 16 & 10 & 6 & 10 & 15 & 13 & 18 & 10 & 10 & 18 & 20 & 22 & 2 & 7 & 12 & 8 \\
\hline DEE & 16 & 10 & 4 & 8 & 11 & 8 & 10 & 10 & 6 & 14 & 14 & 14 & 7 & 2 & 10 & 4 \\
\hline DEF & 24 & 17 & 7 & 10 & 11 & 4 & 20 & 4 & 8 & 17 & 21 & 19 & 12 & 10 & 2 & 11 \\
\hline DEG & 14 & 8 & 6 & 10 & 11 & 10 & 6 & 11 & 6 & 12 & 10 & 11 & 8 & 4 & 11 & 2 \\
\hline$\Sigma$ & 184.5 & 154 & 97 & 133.5 & 138.5 & 117 & 138.5 & 116.5 & 108.5 & 153 & 151 & 153 & 124.5 & 91 & 122.5 & 86.5 \\
\hline
\end{tabular}

Table A.10: External effect matrix for the 16 German states. 


\section{A.2.3 Hydrogen production parameters}

\begin{tabular}{|c|c|c|}
\hline Plant size $\mathrm{d}$ & $\begin{array}{l}\text { Maximum capacity } A_{k} \\
{\left[10^{-3} \mathrm{~m}^{3} \mathrm{hr}^{-1}\right]}\end{array}$ & $\begin{array}{l}\text { Capital cost } c c A_{k} \\
{\left[10^{6} \text { Euro hr } \mathrm{m}^{-3}\right]}\end{array}$ \\
\hline small & 4 & 16.8 \\
\hline medium & 70 & 124.8 \\
\hline large & 500 & 550.8 \\
\hline
\end{tabular}

Table A.11: Maximum capacities and capital cost of the production plants depending on plant size.

\section{A.3 Supporting tables}

\section{A.3.1 Cost allocation}

\begin{tabular}{lccc}
\hline & \multicolumn{3}{c}{ Scenario } \\
Cost [Mio Euro yr & \\
& A & B & C \\
\hline Annualised cost & 5224.5 & 5084.0 & 5480.0 \\
Hydrogen production & 4774.78 & 4773.8 & 4760.71 \\
Hydrogen import & & 3.92 & 56.95 \\
Pipeline investment & 305.65 & 208.83 & 99.62 \\
Pipeline maintenance & 144.07 & 98.43 & 46.95 \\
Production facility investment & & & 350.57 \\
Production facility maintenance & & & 165.24 \\
\hline
\end{tabular}

Table A.12: Cost analysis for scenarios A to C. 


\section{A.3.2 Scenario A}

\begin{tabular}{ccccc}
\hline Inlet & Outlet & $\begin{array}{c}\text { Flow rate } \\
{\left[\mathrm{m}^{3} \mathrm{hr}^{-1}\right]}\end{array}$ & $\begin{array}{c}\text { Diameter } \\
{[\mathrm{cm}]}\end{array}$ & $\begin{array}{c}\text { Velocity } \\
{\left[\mathrm{m} \mathrm{s}^{-1}\right]}\end{array}$ \\
\hline DE1 & DE2 & 452505.61 & 75 & 29.6 \\
DE3 & DE4 & 2656217.73 & 100 & 22.0 \\
DE4 & DE8 & 146906.89 & 50 & 5.2 \\
DE4 & DED & 128847.35 & 50 & 4.4 \\
DE4 & DEE & 2296950.59 & 100 & 20.8 \\
DE5 & DE6 & 45365.23 & 25 & 17.5 \\
DE7 & DE1 & 829010.06 & 75 & 29.3 \\
DE7 & DEB & 771049.44 & 75 & 25.9 \\
DE8 & DEF & 95739.26 & 25 & 18.7 \\
DE9 & DE5 & 62505.05 & 25 & 15.2 \\
DEB & DEA & 586554.11 & 75 & 22.5 \\
DEB & DEC & 37933.83 & 25 & 17.8 \\
DEE & DE9 & 337614.90 & 50 & 14.7 \\
DEE & DEG & 1885029.87 & 100 & 19.8 \\
DEG & DE7 & 1812895.55 & 100 & 23.8 \\
\hline
\end{tabular}

Table A.13: Pipeline diameters, flow rates and velocities in pipelines for scenario A. 


\section{A.3.3 Scenario B}

\begin{tabular}{llccc}
\hline Inlet & Outlet & Flow rate $\left[\mathrm{m}^{3} \mathrm{hr}^{-1}\right]$ & Diameter $[\mathrm{cm}]$ & Velocity $\left[\mathrm{m} \mathrm{s}^{-1}\right]$ \\
\hline DE1 & DE2 & 452505.61 & 50 & 20.1 \\
DE4 & DE3 & 69114.69 & 50 & 10.0 \\
DE4 & DED & 128847.35 & 50 & 21.1 \\
DE6 & DEF & 146353.65 & 50 & 24.5 \\
DE7 & DE1 & 829010.06 & 75 & 12.6 \\
DE7 & DE9 & 483968.56 & 50 & 21.0 \\
DE7 & DEB & 771049.44 & 50 & 27.0 \\
DE7 & DEG & 427915.07 & 50 & 16.1 \\
DE9 & DE5 & 17139.82 & 25 & 9.9 \\
DE9 & DE6 & 191718.88 & 50 & 23.3 \\
DEB & DEA & 586554.11 & 75 & 10.2 \\
DEB & DEC & 37933.83 & 25 & 6.1 \\
DEE & DE4 & 281474.93 & 50 & 22.9 \\
DEF & DE8 & 51167.62 & 50 & 30.0 \\
DEG & DEE & 355780.76 & 50 & 18.9 \\
\hline
\end{tabular}

Table A.14: Pipeline diameters, flow rates and velocities in pipelines for scenario B.

\section{A.3.4 Scenario $\mathrm{C}$}

\begin{tabular}{ccc}
\hline Node & $\begin{array}{c}\text { Capacity } \\
{\left[10^{3} \mathrm{~m}^{3} \mathrm{hr}^{-1}\right]}\end{array}$ & $\begin{array}{c}\text { Hydrogen production } \\
{\left[10^{3} \mathrm{~m}^{3} \mathrm{hr}^{-1}\right]}\end{array}$ \\
\hline DE1 & 500 & 376.5 \\
DE2 & 500 & 452.5 \\
DE7 & 500 & 500 \\
DE9 & 500 & 433.4 \\
DEC & 500 & 475.9 \\
DED & 500 & 479.1 \\
\hline
\end{tabular}

Table A.15: Supply nodes with capacity of production facility for optimised network design. 


\begin{tabular}{ccccc}
\hline Inlet & Outlet & $\begin{array}{c}\text { Flow rate } \\
{[\mathrm{cm}]}\end{array}$ & $\begin{array}{c}\text { Diameter } \\
{\left[\mathrm{m}^{3} \mathrm{hr}^{-1}\right]}\end{array}$ & $\begin{array}{c}\text { Velocity } \\
{\left[\mathrm{m} \mathrm{s}^{-1}\right]}\end{array}$ \\
\hline DE4 & DE3 & 69114.69 & 25 & 9.3 \\
DE4 & DE8 & 51167.62 & 25 & 7.2 \\
DE4 & DED & 200981.67 & 50 & 6.8 \\
DE4 & DEE & 74305.82 & 25 & 10.6 \\
DE5 & DE6 & 58403.95 & 25 & 10.0 \\
DE6 & DEF & 95739.26 & 25 & 24.8 \\
DE7 & DEB & 295198.80 & 50 & 9.8 \\
DE9 & DE5 & 75543.77 & 25 & 10.8 \\
DE9 & DE6 & 82700.54 & 25 & 12.5 \\
DEB & DEA & 586554.11 & 50 & 24.8 \\
DEC & DEB & 437916.80 & 75 & 6.4 \\
DED & DEG & 72134.31 & 25 & 12.3 \\
\hline
\end{tabular}

Table A.16: Pipeline diameters, flow rates and velocities in pipelines for scenario $\mathrm{C}$. 


\section{Nomenclature}

$\begin{array}{ll}\text { Abbreviations } \\ \text { CCS } & \text { carbon capture and storage } \\ \text { EIF } & \text { environmental impact factor } \\ \text { FCV } & \text { fuel cell vehicles } \\ \text { GAMS } & \text { general algebraic modeling system } \\ \text { GIS } & \text { geographical information system } \\ \text { HSC } & \text { hydrogen supply chain } \\ \text { IRF } & \text { inherent risk factor } \\ \text { LCA } & \text { life cycle assessment } \\ \text { MILP } & \text { mixed-integer linear programming } \\ \text { NLP } & \text { non-linear programming } \\ \text { SMR } & \text { steam methane reforming } \\ \text { TRRI } & \text { total relative risk index }\end{array}$

\section{Continuous Variables}

$\bar{\pi}_{i j} \quad$ average pressure in pipeline segment between node $i$ and $j$ $\left[b a r^{2}\right]$

$\hat{I}_{i} \quad$ hydrogen import $\quad\left[m^{3} / h r\right]$

$\lambda_{m 1 i j} \quad$ weighting factor for piecewise linearization of pressure drop between nodes $i$ and $j$, SOS2 variable $\quad[-]$

$\pi_{i} \quad$ squared pressure at node $i \pi_{i} \equiv p_{i}^{2} \quad\left[b_{a r}{ }^{2}\right]$

$\tau_{m 2 i j} \quad$ weighting factor for piecewise linearisation of pressure average between node $i$ and $j$, SOS2 variable $[-]$

CCP pipeline investment cost [Mio.Euro.yr $\left.{ }^{-1}\right]$

FC production facility cost [Mio.Euro.yr $\left.{ }^{-1}\right]$ 


\begin{tabular}{|c|c|c|}
\hline$H C$ & hydrogen production cost & {$\left[\right.$ Mio.Euro.yr $\left.{ }^{-1}\right]$} \\
\hline$I C$ & hydrogen import cost & {$\left[\right.$ Mio.Euro.yr $\left.{ }^{-1}\right]$} \\
\hline$M P$ & pipeline maintenance cost & {$\left[\right.$ Mio.Euro.yr $\left.{ }^{-1}\right]$} \\
\hline$P C$ & pipeline cost & {$\left[\right.$ Mio.Euro.yr $\left.{ }^{-1}\right]$} \\
\hline$Q_{i j}$ & $\begin{array}{l}\text { hydrogen flow rate in pipeline linking node } i \text { a } \\
\text { conditions }\end{array}$ & $\begin{array}{l}\text { and } j \text { at standard } \\
\qquad\left[\mathrm{m}^{3} / \mathrm{hr}\right]\end{array}$ \\
\hline$s E_{i}$ & hydrogen supply at node $i$ & {$\left[m^{3} / h r\right]$} \\
\hline$s l 1_{i j}$ & slack variable for pressure drops & {$\left[b a r^{2}\right]$} \\
\hline$s l 2_{i j}$ & slack variable for pressure average & {$\left[b a r^{2}\right]$} \\
\hline$T C$ & total network cost & {$\left[\right.$ Mio.Euro.yr $\left.{ }^{-1}\right]$} \\
\hline$\psi Y_{d i j}$ & $\begin{array}{l}\text { average pressure in pipeline segment between } \mathrm{r} \\
\text { diameter } d, \psi Y_{d i j} \equiv \sqrt{0.5\left(\pi_{i}+\pi_{j}\right)} Y_{d i j}\end{array}$ & $\begin{array}{r}\text { node } i \text { and } j \text { with } \\
{[\text { bar] }}\end{array}$ \\
\hline$\theta Y_{d i j}$ & $\begin{array}{l}\text { square root of pressure differences (linearised), } \\
{[\text { bar }]}\end{array}$ & $\theta Y_{d i j} \equiv \sqrt{\pi_{i}-\pi_{j}} Y_{d i j}$ \\
\hline$\theta Y_{d i j}$ & $\begin{array}{l}\text { square root of pressure differences (linearized), } \\
\text { [bar }]\end{array}$ & $\theta Y_{d i j} \equiv \sqrt{\pi_{i}}$ \\
\hline
\end{tabular}

\section{Binary Variables}

$E 1_{i} \quad$ establishment of a production facility at node $i$

$E_{k i} \quad$ establishment of a production facility with capacity $k$ at node $i$

$Y_{d i j} \quad$ establishment of a pipeline with diameter $d$ between nodes $i$ and $j$

\section{Parameters}

$\Delta \hat{\pi}_{m 1} \quad$ pressure difference used as intervals in piecewise linearization $\left[b a r^{2}\right]$

$\hat{\Psi}_{m 2} \quad$ pressure average used as intervals in piecewise linearisation $\left[b a r^{2}\right]$

$\hat{\psi}_{m 2} \quad$ square root of pressure average in piecewise linearization [bar $]$ 


\begin{tabular}{|c|c|}
\hline$\hat{\psi}_{m 2}^{\max }$ & maximum of $\hat{\psi}_{m 2}$ \\
\hline$\hat{\psi}_{m 2}^{\min }$ & minimum of $\hat{\psi}_{m 2}$ \\
\hline$\hat{\theta}^{\max }$ & maximum of $\hat{\theta}_{m 1}$ \\
\hline$\hat{\theta}_{m 1}$ & square root of pressure difference in piecewise linearization [bar] \\
\hline$\hat{d}_{d}$ & diameter of pipelines \\
\hline$\pi^{\max }$ & maximum squared pressure \\
\hline$\pi^{\min }$ & minimum squared pressure \\
\hline$\rho_{b}$ & density of hydrogen at standard conditions \\
\hline$a_{0}$ & cost factor for pipelines \\
\hline$a_{12 d}$ & $\begin{array}{l}\text { combination of pipeline cost factors } a_{1} \text { and } a_{2} \text { and diameter } \hat{d}_{d} \\
{[\text { Euro } / \mathrm{km}]}\end{array}$ \\
\hline$a_{1}$ & cost factor for pipelines \\
\hline$a_{2}$ & cost factor for pipelines \\
\hline$A_{k}$ & capacities of production facilities of size $k$ \\
\hline$c c A_{k}$ & $\begin{array}{l}\text { investment cost for production facilities with a capacity of } k \\
{\left[m^{3} / h r\right]}\end{array}$ \\
\hline$c H$ & production cost of hydrogen \\
\hline $\operatorname{crf}$ & capital recovery factor \\
\hline$C U$ & car use per year \\
\hline$d e m_{i}$ & total hydrogen demand of region $i$ \\
\hline$f c$ & fuel consumption \\
\hline$i p$ & cost of imported hydrogen \\
\hline$i t$ & interest rate \\
\hline$k_{p l}$ & coefficient for pressure loss equation \\
\hline$Q_{d}^{\max }$ & $\begin{array}{l}\text { maximum flow allowable in pipelines depending on diameter } d \\
\text { and pressure active in the pipeline } \\
{\left[\mathrm{m}^{3} / \mathrm{hr} / \text { bar }\right]}\end{array}$ \\
\hline
\end{tabular}




$\begin{array}{llr}L_{i j} & \text { distance between regions } & {[\mathrm{km}]} \\ M_{S} & \text { sum of all hydrogen demands } & {\left[\mathrm{m}^{3} \cdot \mathrm{hr}^{-1}\right]} \\ m_{f} & \text { production facility maintenance cost percentage } & {[\%]} \\ m_{p} & \text { pipeline maintenance cost percentage } & {[\%]} \\ n & \text { number of annuities } & {[\mathrm{yr}]} \\ P_{i} & \text { population of region } i & {[\text { people }]} \\ P T & \text { market penetration rate } & {[\%]} \\ Q^{\text {max }} & \text { maximum flow rate allowed by general flow equation } & {\left[\mathrm{m}^{3} / \mathrm{hr}\right]} \\ V O_{i} & \text { vehicle ownership in region } i & {[\text { cars } / \text { person }]}\end{array}$

Sets

$(i, j) \in A \quad$ subset of all possible pipeline connections

$d \in D \quad$ discrete diameter sizes of pipes

$i, j \in N \quad$ supply and demand nodes of sates/regions

$k \in K \quad$ capacities of production facilities

$m 1 \in M 1 \quad$ base points for piecewise linearization of pressure difference

$m 2 \in M 2 \quad$ base points for piecewise linearisation of pressure average

\section{References}

[1] J. Kim, Y. Lee, and I. Moon. An index-based risk assessment model for hydrogen infrastructure. Int. J. Hydrogen Energy, 36(11):6387-6398, 2011.

[2] X. Xu, B. Xu, J. Dong, and X. Liu. Near-term analysis of a roll-out strategy to introduce fuel cell vehicles and hydrogen stations in shenzhen china. Applied Energy, 196:229 - 237, 2017.

[3] J.J. Brey, A.F. Carazo, and R. Brey. Using AHP and binary integer programming to optimize the initial distribution of hydrogen infrastructures in Andalusia. Int. J. Hydrogen Energy, 37(6):5372-5384, 2012. 
[4] B. Gim, Ky. J. Boo, and S. M. Cho. A transportation model approach for constructing the cost effective central hydrogen supply system in Korea. Int. J. Hydrogen Energy, 37(2):1162-1172, 2012.

[5] H. Dagdougui. Models, methods and approaches for the planning and design of the future hydrogen supply chain. Int. J. Hydrogen Energy, 37(6):5318-5327, 2012.

[6] N. Sabio, A. Kostin, G. Guillén-Gosálbez, and L. Jiménez. Holistic minimization of the life cycle environmental impact of hydrogen infrastructures using multi-objective optimization and principal component analysis. Int. J. Hydrogen Energy, 37(6):5385-5405, 2012.

[7] P. Agnolucci and W. McDowall. Designing future hydrogen infrastructure: Insights from analysis at different spatial scales. Int. J. Hydrogen Energy, 38(13):5181-5191, 2013.

[8] S. De-León Almaraz, C. Azzaro-Pantel, L. Montastruc, L. Pibouleau, and O. B. Senties. Assessment of mono and multi-objective optimization to design a hydrogen supply chain. Int. J. Hydrogen Energy, 38(33):14121-14145, 2013.

[9] P. Agnolucci, O. Akgul, W. McDowall, and L. G. Papageorgiou. The importance of economies of scale, transport costs and demand patterns in optimising hydrogen fuelling infrastructure: An exploration with SHIPMod (spatial hydrogen infrastructure planning model). Int. J. Hydrogen Energy, 38(26):11189-11201, 2013.

[10] G. Guillén-Gosálbez, F.D. Mele, and I.E. Grossmann. A bi-criterion optimization approach for the design and planning of hydrogen supply chains for vehicle use. AIChE Journal, 56(3):650-667, 2010.

[11] M. Dayhim, M.A. Jafari, and M. Mazurek. Planning sustainable hydrogen supply chain infrastructure with uncertain demand. Int. J. Hydrogen Energy, 39(13):6789-6801, 2014.

[12] M. Konda, N. Shah, and N. P. Brandon. Optimal transition towards a large-scale hydrogen infrastructure for the transport sector: the case for the Netherlands. Int. J. Hydrogen Energy, 36(8):4619-4635, 2011.

[13] A. Almansoori and N. Shah. Design and operation of a future hydrogen supply chain: snapshot model. Chem. Eng. Res. Des., 84(6):423-438, 2006. 
[14] J.-H. Han, J.-H. Ryu, and I.-B. Lee. Modeling the operation of hydrogen supply networks considering facility location. Int. J. Hydrogen Energy, 37(6):5328-5346, 2012.

[15] S. K. Kamarudin, W. R. W. Daud, Z. Yaakub, Z. Misron, W. Anuar, and NNAN Yusuf. Synthesis and optimization of future hydrogen energy infrastructure planning in Peninsular Malaysia. Int. J. Hydrogen Energy, 34(5):2077-2088, 2009.

[16] A. Almansoori and N. Shah. Design and operation of a future hydrogen supply chain: multi-period model. Int. J. Hydrogen Energy, 34(19):7883-7897, 2009.

[17] P. Nunes, F. Oliveira, S. Hamacher, and A. Almansoori. Design of a hydrogen supply chain with uncertainty. Int. J. Hydrogen Energy, 40(46):16408-16418, 2015.

[18] M. Moreno-Benito, P. Agnolucci, and L. G. Papageorgiou. Towards a sustainable hydrogen economy: Optimisation-based framework for hydrogen infrastructure development. Comput. Chem. Eng., 2016.

[19] N. Johnson, C. Yang, and J. Ogden. A GIS-based assessment of coalbased hydrogen infrastructure deployment in the state of Ohio. Int. J. Hydrogen Energy, 33(20):5287-5303, 2008.

[20] S. Baufumé, F. Grüger, T. Grube, D. Krieg, J. Linssen, M. Weber, J.-F. Hake, and D. Stolten. GIS-based scenario calculations for a nationwide German hydrogen pipeline infrastructure. Int. J. Hydrogen Energy, 38(10):3813-3829, 2013.

[21] A. Hugo, P. Rutter, S. Pistikopoulos, A. Amorelli, and G. Zoia. Hydrogen infrastructure strategic planning using multi-objective optimization. Int. J. Hydrogen Energy, 30(15):1523-1534, 2005.

[22] Z. Li, D. Gao, L. Chang, P. Liu, and E. N. Pistikopoulos. Hydrogen infrastructure design and optimization: a case study of China. Int. J. Hydrogen Energy, 33(20):5275-5286, 2008.

[23] A. Almansoori and A. Betancourt-Torcat. Design of optimization model for a hydrogen supply chain under emission constraints-a case study of Germany. Energy, 111:414-429, 2016. 
[24] J. Kim and I. Moon. Strategic design of hydrogen infrastructure considering cost and safety using multi-objective optimization. Int. J. Hydrogen Energy, 33(21):5887-5896, 2008.

[25] S. De-León Almaraz, C. Azzaro-Pantel, L. Montastruc, and S. Domenech. Hydrogen supply chain optimization for deployment scenarios in the Midi-Pyrénées region, France. Int. J. Hydrogen Energy, 39(23):11831-11845, 2014.

[26] S. De-León Almaraz, C. Azzaro-Pantel, L. Montastruc, and M. Boix. Deployment of a hydrogen supply chain by multi-objective/multi-period optimisation at regional and national scales. Chem. Eng. Res. Des., 104:11-31, 2015.

[27] P. E. Dodds and W. McDowall. A review of hydrogen delivery technologies for energy system models. Technical report, UKSHEC working paper, 2012.

[28] N. Johnson and J. Ogden. A spatially-explicit optimization model for long-term hydrogen pipeline planning. Int. J. Hydrogen Energy, 37(6):5421-5433, 2012.

[29] J. André, S. Auray, J. Brac, D. De Wolf, G. Maisonnier, M.-M. OuldSidi, and A. Simonnet. Design and dimensioning of hydrogen transmission pipeline networks. Eur. J. Oper. Res., 229(1):239-251, 2013.

[30] O. Akgul, A. Zamboni, F. Bezzo, N. Shah, and L. G. Papageorgiou. Optimization-based approaches for bioethanol supply chains. Ind. Eng. Chem. Res., 50(9):4927-4938, 2010.

[31] E. S. Menon. Gas pipeline hydraulics. CRC Press, 2005.

[32] C. Yang and J. Ogden. Determining the lowest-cost hydrogen delivery mode. Int. J. Hydrogen Energy, 32(2):268-286, 2007.

[33] M. Ehrgott. Multicriteria optimization. vol. 491 of lecture notes in economics and mathematical systems, 2005.

[34] R. Beveridge and K. Kern. The Energiewende in Germany: background, developments and future challenges. Renewable Energy L. ES Pol'y Rev., page 3, 2013.

[35] European Commission. Hyways - the european hydrogen roadmap, 2008. 
[36] P. Agnolucci. Hydrogen infrastructure for the transport sector. Int. J. Hydrogen Energy, 32(15):3526-3544, 2007. 